\title{
Endocrine MPA enhances the effects of TAC chemotherapy on improvement of prognosis and increase in long-term survival rates for patients with endometrial cancer
}

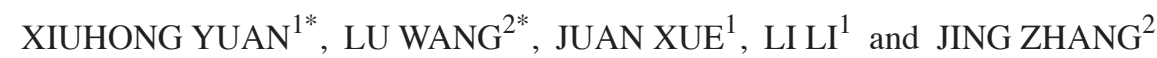 \\ ${ }^{1}$ Department of Gynecology and Obstetrics, The Affiliated Hospital of Yan'an University; \\ ${ }^{2}$ Department of Neurobiology, Medical School of Yan'an University, Yan'an, Shaanxi 716000, P.R. China
}

Received May 28, 2014; Accepted May 26, 2015

DOI: $10.3892 / 01.2015 .3395$

\begin{abstract}
The aim of the present study was to investigate the effect of taxol, adriamycin and carboplatin (TAC) chemotherapy combined with endocrine medroxyprogesterone acetate (MPA) therapy for the treatment of patients with endometrial cancer. A retrospective analysis of 124 patients with endometrial cancer was performed by dividing the cohort into an experimental and control group. The 64 patients in the experimental group received TAC and MPA chemotherapy, whereas the 60 patients in the control group were treated with TAC chemotherapy only. Tissue samples scraped from the uterus were used to extract the total proteins and RNAs for the western blot and reverse transcription-quantitative polymerase chain reaction analyses, respectively. All the patients were followed up for 20-45 months, during which time prognostic data, and one- to three-year survival rates were recorded and compared. The rate of recurrence or metastasis was significantly lower in the experimental group compared with that in the control group $(\mathrm{P}<0.05)$ and the three-year survival rate of the experimental group was significantly higher than that of the control group $(\mathrm{P}<0.05)$. Furthermore, the mean metastasis-associated 1 (MTA1) protein and RNA expression levels were significantly lower in the experimental group compared with the control group $(\mathrm{P}<0.05)$, exhibiting $\sim 30$ and $\sim 15 \%$ of the levels in the control group, respectively. Therefore, a treatment strategy of TAC chemotherapy combined with endocrine MPA therapy appears to effectively improve the prognosis and increase the long-term survival rates of patients with endometrial cancer. Such an enhancing effect may be mediated by the transcriptional downregulation of MTA1 expression.
\end{abstract}

Correspondence to: Dr Jing Zhang, Department of Neurobiology, Medical School of Yan'an University, 38 Guang Hua Road, Yan'an, Shaanxi 716000, P.R. China

E-mail: yxh1586882@163.com

${ }^{*}$ Contributed equally

Key words: taxol, adriamycin, carboplatin, chemotherapy, endocrine therapy, endometrial cancer

\section{Introduction}

Endometrial cancer is a common type of malignant gynecological disease that predominantly occurs in post-menopausal females, aged 50-60 years. It accounts for $20-30 \%$ of cases of female cancer, with the incidence rate demonstrating an increasing trend in recent years (1). Tumors in endometrial cancer patients at clinical stages I/II exhibit a low degree of differentiation and invasive ability, therefore, good results can be obtained using surgical intervention. By contrast, tumors in patients at stages III/IV exhibit a higher degree of malignancy and differentiation, therefore, surgical treatments are only able to reduce tumor volume. Instead, adjuvant endocrine therapy, radiotherapy, and chemotherapy are typically administered for patients at stages III and IV to effectively improve the survival rate (2-4).

The taxol, adriamycin and carboplatin (TAC) chemotherapy regimen has gradually been utilized for the treatment of patients with advanced endometrial cancer. Compared with the traditional adriamycin/cisplatin and cyclophosphamide/adriamycin/cisplatin regimens, the effect of the TAC regimen is more pronounced, causing fewer side effects in patients. In particular, TAC exhibits lower hematological toxicity, thus, resulting in more comprehensive treatment and improved recovery for patients (5).

Endocrine therapy is an effective new method used for the treatment of endometrial cancer. As endometrial cancer cells typically express normal hormone receptors, progesterone-based agents are most commonly used in endometrial cancer treatment strategies; for example, medroxyprogesterone acetate (MPA), megestrol and hydroxyprogesterone (6). Long-term use of progesterone agents can gradually revert the endometrium of endometrial cancer patients back to a healthy state by decreasing the size of the cancer tissues. The predominant action mechanism involves the ability of progesterone to effectively reduce the content of sulfuric acid ester on the cancer cell surface, inhibiting binding between cancer cells and laminin, and, thus, blocking the transfer pathway. Furthermore, progesterone directly inhibits DNA and RNA synthesis, as well as the expression of cancer cells. This results in mature and well-differentiated cells, and accelerates the aging and shrinking of cancerous tissues (7). Previous studies 
have demonstrated that postoperative radiotherapy, chemotherapy and progestin therapy effectively improved the health of high-risk endometrial cancer patients, and prolong their long-term survival rates (5-7). However, the currently employed progestin endocrine therapy has common side effects associated with the long-term use of steroids. For example, progesterone improves patient appetite, and a small number of patients are prone to fluid retention, gastrointestinal discomfort and depression. Additional adverse reactions, such as rare pulmonary embolism, may also occur.

Metastasis-associated protein 1 (MTA1) is a human protein that is encoded by the MTAl gene $(8,9)$. MTA1 is a protein that has been associated with the metastatic state of various cancer types (10-14). MTA1 promotes tumor invasion by the downregulation of E-cadherin in human esophageal squamous cell carcinoma cells (10). Furthermore, it was recently determined that MTA1 promotes the invasion and migration of laryngeal squamous cell carcinoma cells (13). In the present study, 64 patients with endometrial cancer who received TAC chemotherapy combined with endocrine therapy were studied. MTA1 protein and mRNA expression levels were determined, and compared between patients who received TAC chemotherapy only and patients who received TAC plus endocrine therapy.

\section{Patients and methods}

Patients. The present retrospective analysis included 124 patients with endometrial cancer who were hospitalized at the Affiliated Hospital of Yan'an University (Yan'an, China) between January 2011 and April 2013. All the patients were diagnosed by performing a computed tomography scan and biopsy. No patients had received radiotherapy, chemotherapy or endocrine therapy prior to hospitalization. The patients were aged 29-73 years (mean age, $51.4 \pm 6.4$ years) and were classified according to the following clinical stages: Stage I, 58 cases; stage II, 37 cases; stage III, 20 cases; and stage IV, 9 cases. Furthermore, the degree of tumor differentiation within the cohort was as follows: High differentiation, 59 cases; medium differentiation, 42 cases; and low differentiation, 23 cases (15). The patients were divided into an experimental $(n=64)$ and control $(n=60)$ group according to the order of admission. No significant differences in age, clinical stage or differentiation degree were identified between the two groups of patients $(\mathrm{P}>0.05)$ and all the patients were followed up for a period of 20-45 months (mean, $30 \pm 10.4$ months). All procedures were approved by the Ethics Committee of Yan'an University. Informed consent was obtained from all the patients or their family members.

Treatment strategies. Surgical treatment was received by $46 / 64$ patients in the experimental group and 43/60 patients in the control group. Thus, the ratio of patients receiving surgical treatment was similar in the two groups. All the patients were administered the TAC chemotherapy regimen (160 mg/m $\mathrm{m}^{2}$ taxol, $45 \mathrm{mg} / \mathrm{m}^{2}$ adriamycin and AUC5 carboplatin) for 2-8 cycles (cycle duration, 21 days; average number of cycles, $5.3 \pm 1.2$ cycles). Patients in the experimental group were concurrently treated with the progesterone agent MPA (dose, $250 \mathrm{mg} /$ day).
Evaluation index. The patients were scanned with computed tomography three months after treatment and followed up every six months. The recurrence and metastasis evaluation criteria was as follows: i) Local recurrence, the tumor appeared at the pelvic or vaginal stump; and ii) distant metastasis, distant organ metastasis and lymph node metastasis outside of the primary tumor the region (3). Furthermore, survival evaluation was conducted using the following criteria: i) Tumor-free survival period, no reoccurrence or metastasis identified between the termination of the treatment regimen and the termination of the follow-up period; and ii) overall survival period, from the termination of the treatment regimen to the date of patient mortality. Blood cell count, liver and kidney function, urine analysis and physical examinations were performed every week during the treatment and follow-up period.

Biopsy. The patients laid on the examination table with their feet in stirrups for the pelvic biopsy. A speculum was inserted into the vagina to spread the walls of the vagina and expose the cervix. The cervix was then cleansed with an antiseptic solution and a tenaculum, a type of forceps, held the cervix steady for the biopsy. The biopsy curette was inserted into the uterine fundus and, with a scraping and rotating motion, a small quantity of tissue was removed. The removed tissue was placed in formalin or equivalent for preservation. The tissue was sent to a laboratory for processing and analysis, prior to microscopic analysis by a pathologist to provide a histological diagnosis.

Western blot analysis. Total proteins were prepared from tissue samples scraped from the wall of the uterus, as described above. The total proteins were separated using $10 \%$ SDS-polyacrylamide gel electrophoresis and then transferred onto polyvinylidene fluoride membranes (EMD Millipore, Billerica, MA, USA). Following incubation with the polyclonal goat anti-human MTA1 primary antibody (sc-9445; dilution, 1:200; Santa Cruz Biotechnology, Inc., Santa Cruz, CA, USA) at $4^{\circ} \mathrm{C}$ overnight. The membranes were rinsed with Tris-buffered saline and Tween-20, and then incubated with a secondary antibody conjugated to peroxidase (dilution, 1:5,000; Santa Cruz Biotechnology, Inc.) for $1 \mathrm{~h}$. After washing three times, the membranes were detected by enhanced chemiluminescence (EMD Millipore), with GAPDH used for normalization. Furthermore, the relative intensity of the target bands was analyzed by Quantity One 1-D analysis software (Bio-Rad Laboratories, Hercules, CA, USA). Each assay was independently repeated three times.

Reverse transcription-quantitative polymerase chain reaction $(R T-q P C R)$. Total RNA was isolated using TRIzol reagent (Invitrogen Life Technologies, Carlsbad, CA, USA) and mRNA was reverse transcribed to complementary DNA using the Reverse Transcriptase MMLV kit (Takara Biotechnology Co., Ltd., Dalian, China), according to the manufacturer's instructions. PCR reactions were performed using the SYBR ${ }^{\circledR}$ PrimeScriptRT-PCR kit (Takara Biotechnology Co., Ltd.), with relative levels of MTA1 mRNA normalized to GAPDH mRNA. The primer sequences for MTA1 and GAPDH detection were as follows: Forward, 5'-AGCTACGAGCAGCACAACGGGGT-3' and reverse, 5'-CACGCTTGGTTTCCGAGGAT-3' for MTA1; 
Table I. Comparison of recurrence or metastasis between the experimental and control groups.

\begin{tabular}{lccrr}
\hline Group & Cases, $n$ & $\begin{array}{c}\text { Recurrence } \\
\text { or metastasis, } n\end{array}$ & $\begin{array}{c}\text { Time of recurrence } \\
\text { or metastasis, months }\end{array}$ & $\begin{array}{r}\text { Total recurrence } \\
\text { or metastasis rate, } \%^{\mathrm{b}}\end{array}$ \\
\hline Experimental & 64 & 3 & $22.4 \pm 15.4$ & 4.69 \\
Control & 60 & 7 & $20.5 \pm 14.6$ & 11.67 \\
\hline
\end{tabular}

${ }^{\mathrm{a}}$ Mean \pm standard deviation; ${ }^{\mathrm{b}} \chi^{2}, 1.2 ; \mathrm{P}<0.05$.
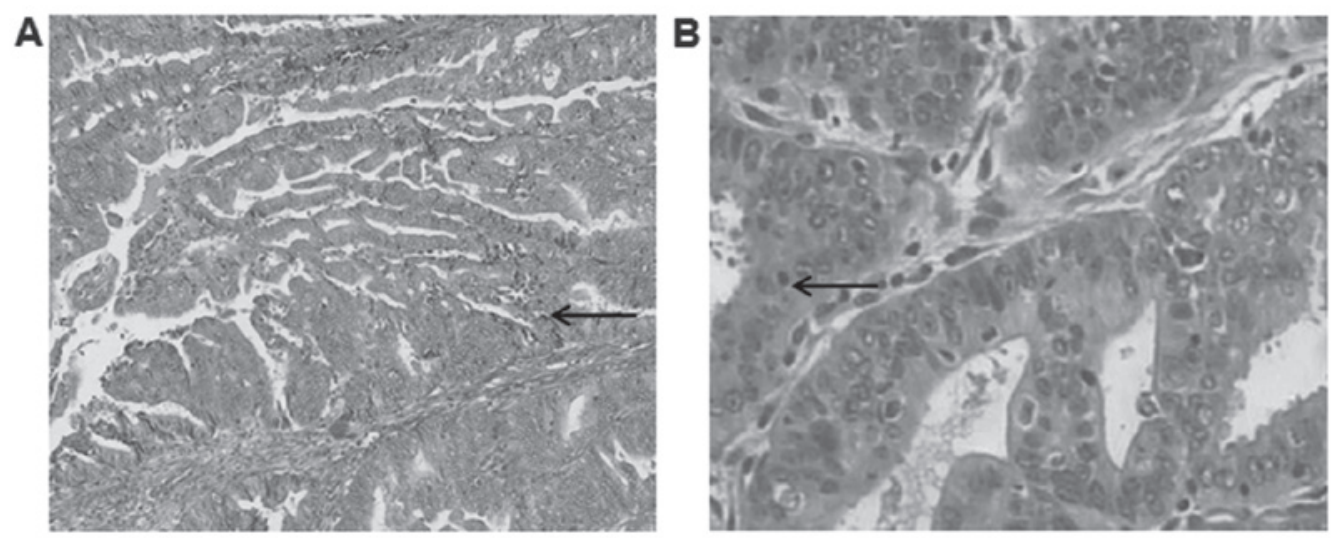

Figure 1. Representative microscopic images of (A) stage I and (B) stage III endometrial cancer biopsies. To perform the biopsy, a biopsy curette was inserted into the uterine fundus. With a scraping and rotating motion, tissue was removed and placed in formalin or equivalent for preservation. The tissue was processed, analyzed and microscopically investigated by a pathologist to provide a histological diagnosis. The samples were stained using hematoxylin and eosin. The magnification was $x$ 400. In (A), the arrow indicates superficial muscle invasions. In (B), the arrow indicates serosa invasions.

and forward, 5'-CATGCGCCTCACTAGTCAGCT-3' and reverse, 5'-TACGCTGAGGATACAGGATAC-3' for GAPDH. qPCR was performed using the ABI 7500 Real-Time PCR system (Applied Biosystems, Foster City, CA, USA) and each experiment was repeated a minimum of three times.

Statistical analysis. SPSS software (version 16.0; SPSS, Inc., Chicago, IL, USA) was used for data analysis. Measurement data was compared using a paired t-test and count data was analyzed by performing a $\chi^{2}$ test. Data are presented as the mean \pm standard deviation and a value of $\mathrm{P}<0.05$ was considered to indicate a statistically significant difference.

\section{Results}

Combination with adjuvant endocrine MPA therapy reduces the recurrence and metastasis rates of patients treated with TAC chemotherapy alone. One hundred and twenty-four endometrial cancer patients were enrolled in the present study, all of whom were diagnosed by performing a computed tomography scan and uterine biopsy (Fig. 1). The patients were divided into two groups, with 64 patients in the experimental group and 60 patients in the control group. The experimental group received TAC and adjuvant endocrine MPA therapy, however, the control group received TAC therapy only. A t-test was performed to compare the recurrence or metastasis rate following treatment between the experimental and control groups. The results indicated that the total recurrence or metastasis rate for the experimental group was significantly decreased compared with the control group $(\mathrm{P}<0.05$; Table I). Furthermore, the time to recurrence or metastasis in the experimental group was significantly prolonged compared with the control group $(\mathrm{P}<0.05$; Table I). Thus, recurrence or metastasis rates and occurrence times following treatment appear to be significantly different between the experimental and control groups.

Combination with adjuvant endocrine MPA therapy improves the long-term survival rates of patients treated with TAC chemotherapy alone. A t-test analysis was performed to compare the one- to three-year survival rates of the experimental and control groups. The one- and two-year survival rates of the patients were not significantly different between the experimental and control groups ( $\mathrm{P}>0.05$; Table II). However, the three-year survival rate of the experimental group was significantly higher than that of the control group $(\mathrm{P}<0.05$; Table II). These results indicate that concurrent treatment endocrine MPA therapy may significantly improve the long-term survival rate of patients treated with TAC chemotherapy.

Adjuvant endocrine therapy with MPA does not cause obvious toxicity in patients. To investigate the toxicity of endocrine therapy, blood, liver and kidney tests, as well as computed tomography scans were performed. The degree of neutropenia, nausea, vomiting and other symptoms in the experimental group were only marginally different from those in the control group (data not shown). In the experimental group, two patients exhibited significant increases in body weight, and one patient developed a cough and asthma, resulting in a $4.69 \%$ 
Table II. Comparison of patient survival rates between the experimental and control groups.

\begin{tabular}{lcccc}
\hline Group & Cases, $\mathrm{n}$ & $\begin{array}{c}\text { One-year } \\
\text { survival rate, } \%^{\mathrm{a}}\end{array}$ & $\begin{array}{c}\text { Two-year } \\
\text { survival rate, } \%^{\mathrm{b}}\end{array}$ & $\begin{array}{c}\text { Three-year } \\
\text { survival rate, } \%^{\mathrm{c}}\end{array}$ \\
\hline Experimental & 64 & 98.2 & 93.4 & 89.4 \\
Control & 60 & 94.5 & 86.5 & 76.1 \\
\hline
\end{tabular}

${ }^{\mathrm{a}} \chi^{2}, 0.39 ; \mathrm{P}>0.05 \cdot{ }^{\mathrm{b}} \chi^{2}, 1.65 ; \mathrm{P}>0.05 .{ }^{\mathrm{c}} \chi^{2}, 3.88 ; \mathrm{P}<0.05$.
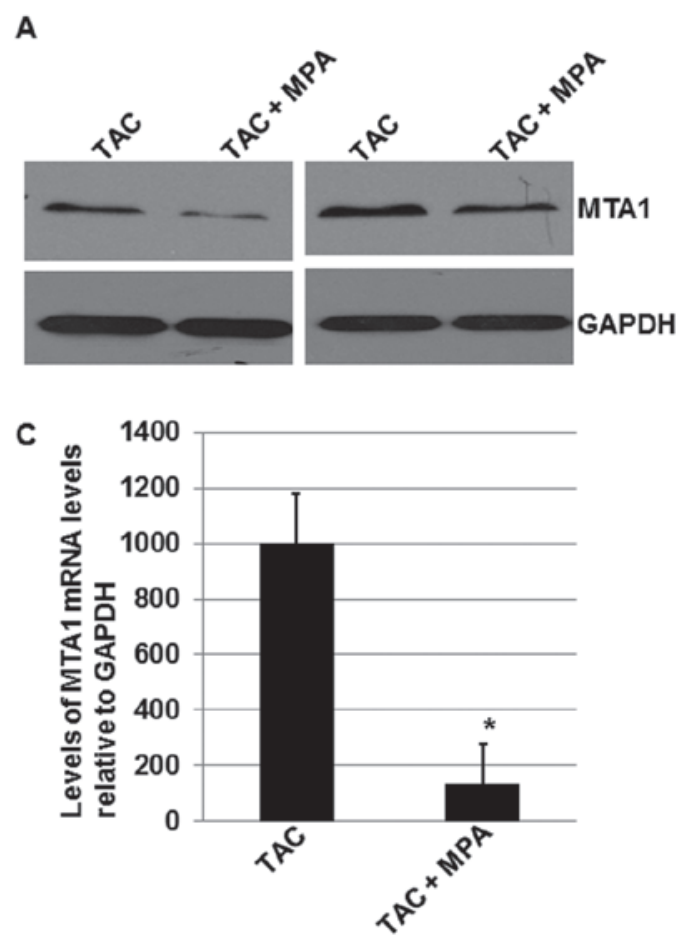

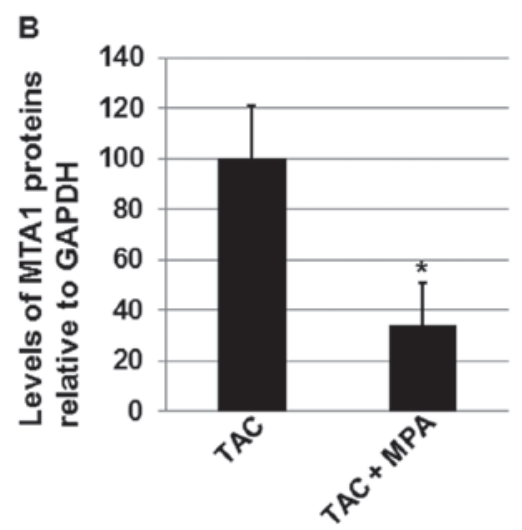

Figure 2. MPA decreases MTA1 protein and RNA levels in human endometrial cancer patients. The tissue samples were scraped off of the uterus wall of the 60 patients in the control group and the 64 patients in the experimental group. (A) The total proteins were extracted for western blot analysis and (B) the relative intensity of the target MTA1 bands were analyzed using Quantity One ${ }^{\circledR}$. Each assay was repeated three times. (C) Total RNA was isolated and analyzed to determine MTA1 mRNA expression levels using reverse transcription-quantitative polymerase chain reaction. Each assay was repeated three times and the error bars indicate the standard deviation from the mean. ${ }^{*} \mathrm{P}<0.05$. TAC, taxol, adriamycin and carboplatin; MPA, medroxyprogesterone acetate; MTA1, metastasis-associated protein 1.

incidence of adverse reactions. The aforementioned adverse reactions were not identified in the control group; however, the difference in adverse reactions between the experimental and control group was not statistically significant $(\mathrm{P}>0.05)$. These data indicate that adjuvant endocrine MPA therapy does not cause significant toxicity in patients with endometrial cancer.

MPA decreases MTA1 expression levels in patients treated with TAC chemotherapy. As demonstrated in the aforementioned results, the MTA1 protein was associated with recurrence and metastasis rates in patients with endometrial cancer. To investigate the mechanism underlying the inhibitory effect of MPA on the recurrence and metastasis rates of patients treated with TAC chemotherapy, the total proteins and RNAs were extracted from tissue samples scraped off of the uterus wall for analysis. Western blot analyses were performed to determine the MTA1 protein expression levels in the 60 control group and 64 experimental group patients (Fig. 2A). As indicated in
Fig. 2B, combined treatment with TCA and MPA significantly enhanced the inhibitory effects of TAC therapy on the expression levels of the MTA1 protein $(\mathrm{P}<0.05)$. Furthermore, the mean MTA1 expression level in the experimental group was $\sim 30 \%$ of the level in the control group. These results indicate that MPA may reduce MTA1 expression levels in patients that receive TAC chemotherapy.

MPA decreases MTA1 expression levels in patients treated with TAC chemotherapy via a transcriptional mechanism. To determine whether the effect of MPA treatment on MTA1 expression is mediated via a translational or transcriptional mechanism, the total RNAs were isolated from the tissue samples scraped off of the uterus wall of all patients in the present study. Using RT-qPCR, it was identified that the mRNA expression levels of MTA1 were significantly affected by treatment with MPA ( $<<0.05$; Fig. $2 \mathrm{C}$ ), with the mean level of MTA1 mRNA $\sim 15 \%$ of the mean level in the control group ( $\mathrm{P}<0.05$; Fig. $2 \mathrm{C}$ ). 
These results indicate that MTA1 expression may be downregulated via a transcriptional mechanism.

\section{Discussion}

By performing adjuvant endocrine therapy on 65 stage I endometrial cancer patients, Xie et al (16) demonstrated that the recurrence and metastasis rates declined in comparison to the patients who did not receive adjuvant endocrine MPA therapy. However, this difference was not significant. In addition, following a treatment period of $>12$ months, the three-year tumor-free survival rate of $100 \%$ was significantly increased compared with the control group. Similarly, the present study identified that the recurrence or metastasis rate of the experimental group was significantly lower than in the control group, and the recurrence or metastasis occurrence time of the experimental group was significantly prolonged compared with the prognosis of the control group. Furthermore, the one-year and two-year survival rates of the experimental group were not significantly different from that of the control group, however, the three-year survival rate of the experimental group was significantly higher compared with that of the control group $(\mathrm{P}<0.05)$. A number of patients in the experimental and control groups exhibited certain degrees of hematologic toxicity following the treatment regime, however, the toxicity responses were not significantly different between the two groups. Therefore, the results of the present study demonstrated that TAC chemotherapy combined with endocrine MPA therapy may effectively improve the prognosis and survival rates of patients with endometrial cancer.

MTA1 is associated with the metastatic state in various types of cancer (9-15). For example, recent studies have demonstrated that MTA1 promotes tumor invasion in human esophageal (11) and laryngeal (14) squamous cell carcinoma cells. In the present study, it was identified that combined use of MPA and TAC chemotherapy may significantly decrease the expression levels of MTA1 in patients with endometrial cancer. In addition, it was determined that this inhibitory effect on MTA1 is mediated in a transcriptional manner.

In conclusion, TAC chemotherapy combined with endocrine MPA therapy appears to effectively reduce the recurrence or metastasis rates, improve the prognosis, and increase the long-term survival rates of patients with endometrial cancer. Furthermore, such an effect may be mediated by downregulation of the expression levels of MTA1 via a transcriptional mechanism.

\section{Acknowledgements}

The present study was supported by Yan'an University (Yan'an, China).

\section{References}

1. Xing X, Li X, Qiao S, et al: Endocrine cells of endometrial cancer and its endocrine differentiation mechanism. Hebei Med J 32: 3542-3544, 2010 (In Chinese).

2. Wang J: Scientific understanding of standard treatment for endometrial cancer. J Zhengzhou Univ (Med Sci) 47: 1-3, 2012 (In Chinese).

3. Cao Z: Common gynecological cancer treatment guidelines. People's Health Publishing House, Beijing, pp41-45, 2007.

4. Wang $J$ and Wei L: New Progress in the adjuvant treatment of endometrial cancer. Chinese J Obstet Gynecol 3: 156-158, 2004.

5. Cui M and Xu T: The research progress of endometrial cancer. Prac J Oncol 27: 202-205, 2013.

6. Li X: Clinical diagnosis and treatment of endometrial cancer. Guangdong Med J 33: 1185-1187, 2012 (In Chinese).

7. Zhu Y and Gao Q: Progestin therapy for endometrial cancer. Chinese J Prac Gynecol Obstet 18: 204-205, 2002 (In Chinese).

8. Toh Y, Pencil SD and Nicolson GL: A novel candidate metastasis-associated gene, mtal, differentially expressed in highly metastatic mammary adenocarcinoma cell lines. cDNA cloning, expression and protein analyses. J Biol Chem 269: 22958-22963, 1994.

9. Toh Y, Pencil SD and Nicolson GL: Analysis of the complete sequence of the novel metastasis-associated candidate gene, mta1, differentially expressed in mammary adenocarcinoma and breast cancer cell lines. Gene 159: 97-104, 1995.

10. Weng W, Yin J, Zhang Y, Qiu J and Wang X: Metastasis-associated protein 1 promotes tumor invasion by downregulation of E-cadherin. Int J Oncol 44: 812-818, 2014.

11. Kang HJ, Lee MH, Kang HL, et al: Differential regulation of estrogen receptor $\alpha$ expression in breast cancer cells by metastasis-associated protein 1. Cancer Res 74: 1484-1494, 2014.

12. Bettum IJ, Vasiliauskaite K, Nygaard V, et al: Metastasis-associated protein S100A4 induces a network of inflammatory cytokines that activate stromal cells to acquire pro-tumorigenic properties. Cancer Lett 344: 28-39, 2014.

13. Zhang H, Yang D, Wang H, et al: Metastasis-associated gene 1 promotes invasion and migration potential of laryngeal squamous cell carcinoma cells. Oncol Lett 7: 399-404, 2014.

14. Nagaraj SR, Shilpa P, Rachaiah K and Salimath BP: Crosstalk between VEGF and MTA1 signaling pathways contribute to aggressiveness of breast carcinoma. Mol Carcinog: Nov 22, 2013 (Epub ahead of print).

15. Hoffman BL, Schorge JO, Schaffer JI, Halvorson LM, Bradshaw KD, Cunningham FG and Calver LE (eds.): Endometrial Cancer. In: Williams Gynecology. 2nd edition. McGraw-Hill, New York, NY, pp 825, 2012.

16. Xie L: A clinical analysis of the effects of adjuvant endocrine therapy for stage I endometrial carcinoma. J Clin Exp Med 12: 1063-1064, 2013. 